\title{
Pitch Perturbation of Sonorants and a Three-way Tonal Split in Chinese
}

\author{
KENING LI \\ University of Washington
}

\section{Introduction}

In this paper, I would like to discuss a three-way tonal split in the history of the Chinese language, for which there is still no satisfactory explanation, and then provide an explanation from a phonetic perspective, which mostly concerns pitch perturbation of sonorant consonants.

\section{A Three-way Tonal Split from Middle Chinese to Old Mandarin}

The tonal change the current paper is concerned with happened in the period from Middle Chinese (hereafter "MC") to Old Mandarin (hereafter "OM"). According to a common view of periodicization of the Chinese language (Norman 1988), $\mathrm{MC}$ refers to the language from approximately $450 \mathrm{AD}$ to $1300 \mathrm{AD}$ and in particular is taken to be represented by the language recorded in the famous rhyme book Qieyun compiled in 601AD. OM refers to the Mandarin dialect in the period from approximately $1300 \mathrm{AD}$ to $1650 \mathrm{AD}$. $\mathrm{OM}$ is generally considered a direct descendent of MC.

As is generally believed, there were four distinctive tones in $\mathrm{MC}$ and in $\mathrm{OM}$ as well. But they were not in a relationship of one-to-one correspondence. Instead, there was a series of complicated tonal splits and mergers that happened to the four MC tones, which turned into four new tones in OM. I will call the four MC tones Tone A, B, C and D, respectively, and the four OM tones Tone 1, 2, 3 and 4 , respectively, in the following sections. What concerns us here is only the split that happened to MC Tone $\mathrm{D}$, so I will leave out discussions of the other three $\mathrm{MC}$ tones for simplicity. ${ }^{1}$

MC Tone D underwent a three-way tonal split, as conditioned by the manner of articulation of syllable onset consonants. Syllables beginning with a voiceless

\footnotetext{
${ }^{1}$ The complete picture for categorical tonal changes from $\mathrm{MC}$ to $\mathrm{OM}$ is: $\mathrm{MC}$ Tone A split into OM Tone 1 and 2, MC Tone B split into OM Tone 3 and 4, no split happened for MC Tone C, which merged into OM Tone 4, and lastly, MC tone D split in three ways and merged into OM Tones 2, 3 and 4 respectively.
} 


\section{Kening Li}

obstruent changed to bear OM Tone 3, syllables beginning with a voiced obstruent changed to bear OM Tone 2, and syllables beginning with a (voiced) sonorant changed to bear OM Tone 4. This process is schematized as follows:

(1) A three-way split of Tone D from MC to OM. $p$ stands for all voiceless obstruent onsets, $b$ stands for all voiced obstruent onsets and $m$ stands for all sonorant onsets.

A

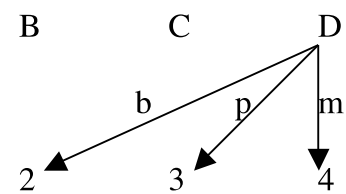

MC Tones

\section{OM Tones}

It is worth noting that although the categorical changes between $\mathrm{MC}$ tones and $\mathrm{OM}$ tones are agreed on by most people working on Chinese historical phonology, unfortunately there is only very limited agreement on, or knowledge of, the exact phonetic values of these tones.

The three-way tonal split found in Chinese is very unique and seems to be unattested in any other language. So far there have not been any satisfactory explanations for it.

\subsection{Tonogenesis Theory and Pitch Perturbation}

The tonal split described above should immediately remind us of tonogenesis theory, which deals with how tones originate and change in language. It is based on phonetic grounds, more specifically, theories of pitch perturbation, which are about how consonants influence the pitch of adjacent vowels. The best-known case of tonogenesis is a two-way tonal split conditioned by the voicing feature of syllable onset consonants. It has been found in acoustic studies that voiceless obstruents significantly raise the pitch $\left(\mathrm{F}_{0}\right)$ of the onset of a following vowel, whereas voiced obstruents significantly lower the pitch of the onset of a following vowel (Hombert, et al. 1979; Ohala 1972, 1974). Based on these acoustic findings in pitch perturbation, tonogenesis theory states that when a voicing contrast on obstruents exists, the concomitant pitch difference is only an allophonic variation; when the voicing distinction is lost, the previously existing pitch difference becomes phonologized, and thus two contrastive tones emerge. When such a development occurs, a relatively lower tone develops on vowels following a previously voiced series, and a relatively higher tone develops on vowels following a previously voiceless series (Matisoff 1973; Hombert, et al. 1979; Li 1980; Svantesson 1989; among others).

Obviously, the three-way tonal split that happened to Tone D from MC to OM can be partially explained by the tonogenesis theory sketched above, namely, two of the three split paths that were conditioned by the voicing feature of obstruent onsets. The fact that sonorants alone conditioned a separate split path in the three- 
way tonal split seems to suggest that sonorants have a distinct pitch perturbation property from either type of obstruents.

Unlike the largely consistent results regarding the pitch perturbation properties of obstruents, results from previous studies concerning sonorants' effect on $F_{0}$ did not always agree with each other.

In an acoustic study conducted by Christovich (1969), he measured the intraoral pressure $\mathrm{P}_{0}$ and the fundamental frequency $\mathrm{F}_{0}$ of the intervocalic $[\mathrm{m}]$ and $[\mathrm{b}]$ in words "mama" and "baba" during monotone utterances. He found that in the stop interval for "baba," $\mathrm{P}_{0}$ increases and $\mathrm{F}_{0}$ decreases, while in the stop interval for "mama," there is no increasing of $\mathrm{P}_{0}$ nor decreasing of $\mathrm{F}_{0}$, because "the air escapes freely to the outside through the nasal openings" (p. 372). These findings seem to predict that sonorants should have a different effect from voiced obstruents on the following pitch.

However, in Hombert, et al. (1979), sonorants are found to behave similarly to voiced stops for some speakers in their study, depressing the pitch of the beginning of a following vowel. Interestingly, the authors were somewhat surprised by this lowering effect of sonorants, as they commented on sonorants as a group of consonants "which one would expect to perturb $\mathrm{F}_{0}$ minimally or not at all (since they should not generate any oral pressure build-up)" (p. 40). There is an important detail in their experiment that I think might be responsible for the apparent lowering effect and that was also noticed by the authors. This detail is that the intonation contour with which the target words were spoken was rise-fall. It is important because this particular intonation contour may be the real source for the apparent F0 lowering after sonorants. The real pitch perturbation effect of sonorants was disguised by the uncontrolled intonation factor.

Another study that dealt with pitch perturbation of sonorants is Maddieson (1984) with Burmese. Burmese is a tonal language with a phonemic contrast between voiced and voiceless nasals and laterals. The acoustic data show that the voiced sonorants lower the following pitch and their voiceless counterparts raise it, so the first part agrees with the findings of Hombert, et al. (1979). Maddieson concludes that voiced vs. voiceless sonorants have pitch perturbation effects parallel to those of voiced vs. voiceless obstruents. However, I think there could be other explanations for the results. As we know, voicing contrast on sonorants is auditorily not salient; therefore, there may be some other accompanying phonetic cues that are imposed upon the sonorants to enhance the perceptual distinction. If this accompanying phonetic cue is a pitch difference on the following vowel, then the different pitch is just a phonological effect, not phonetic; if this accompanying phonetic cue is something like phonation type, then the phonation type may be the real source for the pitch perturbation.

Due to the existence of some possible uncontrolled factors in previous studies and inconclusiveness of previous findings regarding sonorants, it is necessary to conduct another acoustic experiment to find out the exact nature of pitch perturbation properties of sonorants. The following section discusses such an experiment. 


\section{Kening Li}

\section{An Acoustic Study: Pitch Perturbation of Sonorants in Mandarin}

Based on the examination of previous studies, I hypothesize that (voiced) sonorants do not have a significant effect on the $F_{0}$ of a following vowel, i.e. they neither raise it nor lower it. To test this hypothesis, I conducted an acoustic experiment with Mandarin Chinese.

\subsection{Experiment Design and Methodology}

Five native speakers of Mandarin from Beijing, 2 male and 3 female, were chosen to participate in a recording session. They were asked to read some monosyllabic words in isolation written in Chinese characters from a reading list. The list is attached in the appendix following the main text. Monosyllabic words in isolation instead of in sentence frames were used because tones on adjacent syllables will significantly influence each other (Shen 1990, Xu 1997), and thus obscure the real source of pitch perturbation. All chosen syllables bear the structure $\mathrm{CV}$, where the $\mathrm{V}$ is constantly [a] to eliminate the possible $\mathrm{F}_{0}$ variation caused by different vowel qualities (Ohala 1973). Six onset consonants were chosen: [p, t, k, m, n, l]. The three sonorants are the focus of the experiment. Three voiceless unaspirated stops were incorporated for comparison purposes. Note that there are no voiced obstruents anymore in modern Mandarin. All chosen syllables bear the same tone, the high level tone, because of its flat contour and stable value. The other three lexical tones in Beijing Mandarin are contour tones and thus were eliminated from the experiment in order to factor out the influence of intonation (here, meaning the pitch contour intrinsic to each tone). All characters were read with four repetitions with random ordering. There are $4 * 6 * 5=120$ tokens in total for examination.

The data collected were then subjected to pitch analysis with Praat with a sampling rate of $44 \mathrm{kHz} . \mathrm{F}_{0}$ was measured in every syllable for the first $200 \mathrm{~ms}$ from the vowel onset at intervals of $10 \mathrm{~ms}$. In total, 21 measurements of $F_{0}$ were recorded for each syllable. As for the determination of vowel onset, for syllables beginning with onset $[\mathrm{p}],[\mathrm{t}]$ or $[\mathrm{k}]$, the measurement starts from the voicing onset, and for syllables beginning with onset [m], [n] or [1], the measurement starts at a point where the formants transition between the sonorant and the following vowel. Some degree of subjectivity in deciding where that point is was unavoidable.

\subsection{Results}

After normalizing all the measurements, it is found that syllables beginning with a voiceless stop fall into one pattern and syllables beginning with a sonorant fall into another, just as expected. $\mathrm{F}_{0}$ data for the former keep dropping until around $80 \mathrm{~ms}$ and then become stabilized after that, whereas for the latter, $F_{0}$ value is pretty much the same from the beginning to the end with slight variation here and there. $\mathrm{F}_{0}$ data after these two types of consonants averaged over all repetitions and all speakers are mapped into pitch curves as shown below in (2) and (3). 
(2) $F_{0}$ frequency by time: Vowels after voiceless stops

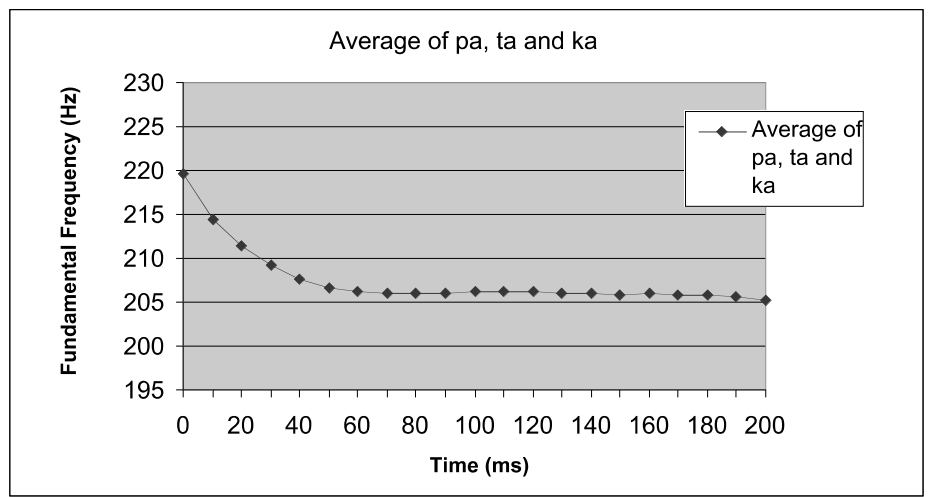

(3) $F_{0}$ frequency by time: Vowels after sonorants

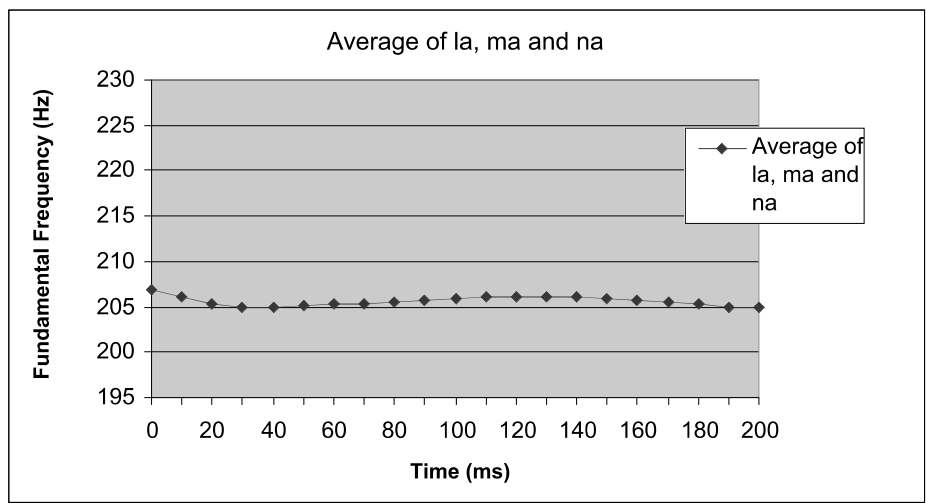

In (2), the $F_{0}$ difference between the vowel onset and 80ms (approximately where the raising effect of voiceless stops ends) is $13.73 \mathrm{~Hz}$. In contrast, in (3), there is no obvious turning point on the pitch curve. The $\mathrm{F}_{0}$ difference between the vowel onset and the lowest point of the first $200 \mathrm{~ms}$ (around $40 \mathrm{~ms}$ ) is $1.925 \mathrm{~Hz}$, which can be considered trivial.

To show the contrast better, the above two pitch curves are mapped together in one picture in (4). 


\section{Kening Li}

(4) Comparison of $F_{0}$ frequency by time: Vowels after voiceless stops and sonorants

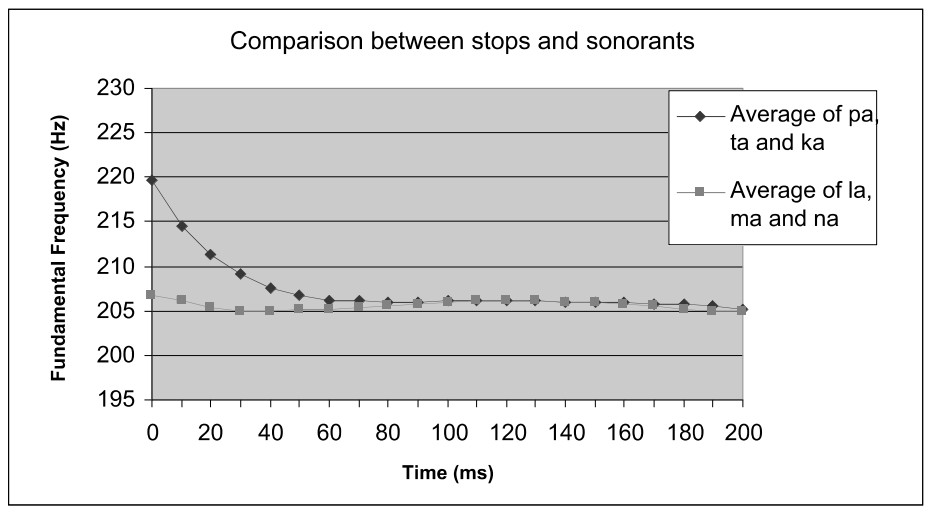

\subsection{Conclusions}

Based on the results, the following conclusions can be drawn for Mandarin Chinese. Voiceless stops [p, t, k] raise the onset pitch of a following vowel significantly, confirming previous studies. Sonorants (in this case, nasals and a liquid) do not exert any significant effect on the onset pitch of a following vowel, i.e. they neither raise nor lower the pitch, supporting my hypothesis.

\section{Explanation for the Three-way Tonal Split}

\subsection{Main Proposal}

Now let us come back to the original problem of the three-way tonal split in Chinese. To remind the readers, the pattern of the three-way split is repeated below.

(5) A three-way split of tone D from MC to OM. $P$ stands for all voiceless obstruent onsets, $b$ stands for all voiced obstruent onsets and $m$ stands for all sonorant onsets.

A

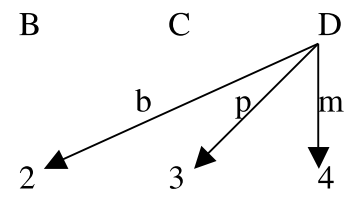

MC Tones

1

2

3

OM Tones

Based on the new finding regarding pitch perturbation of sonorants and previous findings on obstruents, I propose the following explanation for the three-way tonal split:

The three-way split of Tone D in MC is due to the three distinct pitch pertu rbation properties of three types of consonant onsets: a voiceless obstruent signifi- 
cantly raises the pitch of the onset of a following vowel, a voiced obstruent significantly lowers the pitch of the onset of a following vowel, and a (voiced) sonorant neither significantly raises nor significantly lowers the pitch of a following vowel.

In other words, each of the three types of consonants affects the following pitch in a different way, so when the previously allophonic pitch difference in MC Tone $\mathrm{D}$ was phonologized by the time of $\mathrm{OM}$, one tone split into three tones.

\subsection{A Follow-up Question}

The above immediately analysis gives rise to a new problem. The analysis predicts that every MC tone should undergo a three-way split since the same kinds of pitch perturbation effects affected them. Yet the historical fact is that among the four MC tones only Tone D ended up splitting up three ways. MC Tone A and B each underwent a two-way split and there was no split at all for Tone C. How to explain this?

After a closer look at Tone D, I find that Tone D syllables have certain unique properties that may contribute to the fact that Tone D was the only tone that underwent a three-way split.

Unlike MC Tones A, B and C, which were distinguished purely by distinctive pitch contours, Tone D syllables distinguished themselves also by a unique syllable structure. They always ended with one of the three voiceless and probably unreleased stops [p, t, k], whereas syllables bearing the other three tones ended either with a vowel or with a nasal. This different structure of Tone D syllables is important because it was accompanied by certain other features, which in turn would influence the pitch perturbation effects upon them. These accompanying features are their abruptness and short duration. In the literature of Chinese historical phonology, tones like MC Tone D are referred to as "entering" tones or "checked" tones.

In some contemporary descriptions of MC, Tone D was described as "short and abrupt" (Chu 806-827). In two modern works reconstructing MC tones, Tone $\mathrm{D}$ is reconstructed as "shorter than usual length and abrupt" yet "with uncertain pitch contour" (Mei 1970, Ting 1995). There are a number of modern Chinese dialects that still preserve entering tones, such as Cantonese, Min, and Hakka. The entering tones in these dialects are unanimously described as "short" tones (Dictionary of Modern Chinese Dialects, 1993-1995). Their shortness is additionally supported by two studies based on actual recordings in Cantonese. Zee (1991) states that in Cantonese, certain vowels are "long in open syllables or syllables closed by a nasal, and half-long in syllables closed by a plosive," and certain vowels are "short in syllables closed by a nasal and extra-short in syllables closed by a plosive." In Lee, Kochanski, Shih and Li (2002), entering tones in Cantonese are described as "contrastingly shorter in duration than the non-entering tones." In a schema given in the second study, the duration of an entering tone is less than half of the duration of a non-entering tone. 


\section{Kening Li}

Under the assumption that MC Tone D syllables were abrupt and much shorter than other tones, I would like to propose the following to account for the fact that the three-way tonal split only happened to Tone D:

For MC Tone D (which is short and abrupt), pitch perturbation effects of the onset consonants range over the entire syllable or most of it. This results in three more distinct pitch contours that are more likely to stay distinct from each other, whereas for the other MC tones (which are of normal length), the pitch perturbation effects only reach the onset of the following vowel and thus may not alter the overall pitch contours in a significant way. Some of the resulting allotones with closer pitch levels are likely to merge due to possible perceptual reasons.

The exact nature of the possible perceptual reasons are not clear to me at this point. The contrast between the two different pitch perturbation effects on entering vs. non-entering tones is schematized as follows in (6).

(6) A schema showing the possible different pitch perturbation effects on entering and non-entering tones. The two horizontal lines at the bottom indicate duration.

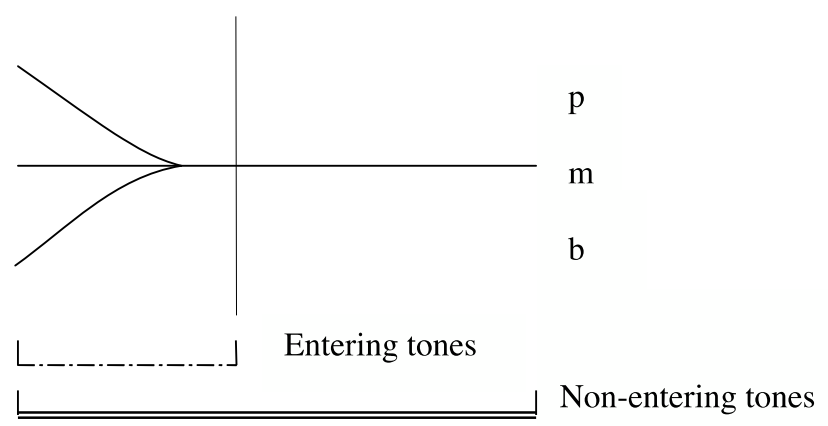

In (6), the entering tone is represented as slightly less than half as long as the non-entering tone. If the absolute duration of the pitch that is affected by prevocalic consonants is the same for both types of tones, the relative portion that is affected in the entering tone will be much greater than that in the non-entering tone. Therefore, the resulting three allotones of an entering tone will be perceptually more distinct from each other to speakers. In contrast, the resulting three allotones of a non-entering tone are perceptually closer to each other. Thus they are less likely to stay distinct, and two or even all three of them may end up merging back into one tone. This was proposed to be the case with the other three $\mathrm{MC}$ tones in $\mathrm{Li}$ (2002).

\section{Conclusions}

This paper examines a unique three-way tonal split that happened to one particular tonal category-MC Tone $\mathrm{D}$-in the evolution from $\mathrm{MC}$ to $\mathrm{OM}$ in the history 
of the Chinese language. An explanation of this tonal split is proposed from a phonetic perspective. The major points are summarized below.

In a phonetic experiment I conducted with Mandarin Chinese, sonorants are found to exert neither a significant raising nor a significant lowering pitch perturbation effect on the pitch of a following vowel. This finding, combined with previous findings that voiceless obstruents significantly raise the pitch of the onset of a following vowel and their voiced counterparts significantly lower it, will account for the three-way tonal split in a straightforward fashion. In addition, the unique syllable structure of MC Tone D (an entering tone), in particular its accompanying abruptness and shortness, explain why the three-way split only happened to Tone $\mathrm{D}$, but not to the other three tones in MC.

Although the current paper only investigates one specific phonological change in one language, it has presented an example of how universal phonetic rules can play an important role in accounting for difficult problems in historical phonology. This implication can certainly be generalized to a much wider scope in modern linguistics.

\section{Future Research}

This paper suggests to us at least the following directions for future research. First, it is desirable to apply the experiment above to more speakers in Mandarin and to more languages. Second, to confirm the idea that prevocalic consonants will affect the pitch of a larger proportion of the vowel for entering tones than for non-entering tones, further phonetic studies need to be done.

\section{Appendix}

The list of Mandarin words in the acoustic experiment:

[pa] 'eight'

[ta] 'put up, erect (among many other meanings)'

[ka] 'quack'

[ma] 'mother'

[na] intonation particle

[la] 'pull'

\section{References}

Chistovich, L.A. 1969. Variation of the fundamental voice pitch as a discriminatory cue for consonants. Soviet Physics-Acoustics 114(3).

Chu, Zhong. 806-827 AD. Yunhe yunpu, now lost.

Hombert, Jean-Marie. 1977. Consonant types, vowel height and tone in Yoruba. Studies in African Linguistics 8(2).

Hombert, Jean-Marie, John Ohala, and William G. Ewen. 1979. Phonetic explanation for the development of tones. Language 55(1). 


\section{Kening Li}

Lee, Tan, Greg Kochanski, Chilin Shih and Yujia Li. 2002. Modeling tones in continuous Cantonese speech. Proceedings of the International Conference on Spoken Language Processing, Denver, Colorado.

Li, Fang-Kuei. 1980. Laryngeal features and tone development. Chungyang yenchiu yuan lishih yuyen yenchiuso chik'an 51.

Li, Kening. 2002. Pitch Perturbation and its Implications for the Tonal Development from Middle Chinese to Old Mandarin. Master thesis, University of Washington, Seattle.

Maddieson, Ian 1984. The effects on $\mathrm{F}_{0}$ of a voicing distinction in sonorants and their implications for a theory of tonogenesis. Journal of Phonetics 12.

Matisoff, James A. 1973. Tonogenesis in Southeast Asia. In L. Hyman, ed., Consonant Types and Tone. L.A.: Linguistics Program, USC.

Mei, Tsu-lin. 1970. Tone and prosody in Middle Chinese and the origin of the rising tone. Harvard Journal of Asiatic Studies 30:86-110.

Norman, Jerry. 1988. Chinese. Cambridge University Press.

Ohala, J. 1972. How is pitch lowered? JASA 52.134.

Ohala, J. 1973. Explanations for the intrinsic pitch of vowels. Monthly Internal Memorandum (Jan.), 9-26. Phonology Lab, University of Calif., Berkeley.

Ohala, J. 1974. Experimental historical phonology. In A. Bruck, R.A. Fox, and M.W. LaGaly, eds., Historical Linguistics II: Theory and Description in Phonology. Proceedings of the $1^{\text {st }}$ International Conference on Historical Linguistics, Edinburgh. Amsterdam: North Holland.

Shen, Xiaonan Susan. 1990. Tonal coarticulation in Mandarin. Journal of Phonetics 18(2):281-95.

Svantesson, Jan-Olof. 1989. Tonogenetic mechanisms in Northern Mon-Khmer. Phonetica 46.

Ting, Pang-hsin. 1995. Tonal evolution and tonal reconstruction in Chinese. New Horizons in Chinese Linguistics. Kluwer Academic Publishers.

Xiandai hanyu fangyan da cidian. [Dictionary of Modern Chinese Dialects.] 1993-1995. Nanjing: Jiangsu Education Press.

$\mathrm{Xu}, \mathrm{Yi}$. 1997. Contextual tonal variations in Mandarin. Journal of Phonetics 25(1): 61-83.

Yip, Moira. 1990. The Tonal Phonology of Chinese. Garland Publishing, Inc.

Yip, Moira. 2002. Tone. Cambridge: Cambridge University Press.

Zee, Eric. 1991. Ilustrations of the IPA-Chinese: Hong Kong Cantonese. Journal of IPA 21(1):46-48.

Kening Li

University of Washington

Department of Linguistics

Box 354340

Seattle, WA $98195-4340$

kening@u.washington.edu 